\title{
XLVI. On method in causal research
}

\section{S. Tolver Preston}

To cite this article: S. Tolver Preston (1880) XLVI. On method in causal research , Philosophical Magazine, 9:57, 356-367, DOI: 10.1080/14786448008626853

To link to this article: http://dx.doi.org/10.1080/14786448008626853

曲 Published online: 28 Apr 2009.

Submit your article to this journal $\pi$

Џ Article views: 5

Q View related articles $₫$

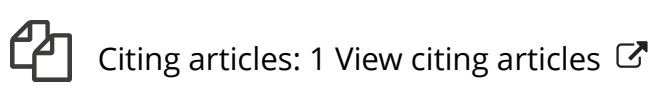


A single circuit was arranged as shown in fig. 3.

Fig. 3.

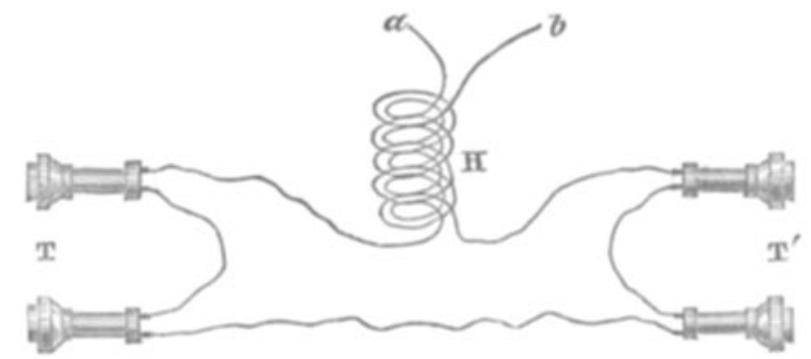

$\mathrm{T}$ and $\mathrm{T}^{\prime}$ are the telephones, $\mathrm{H}$ is the double helix. The helix was joined up as shown in the figure, one end of each of its wires, $a$ and $b$, being left unconnected and insulated. Thus arranged, there was a break in the continuity of the circuit, but nevertheless speech was transmitted with perfect distinctness, and conversation carried on with the same facility as in the previous experiments, the helix acting not as a conductor, but as a condenser, its two wires replacing the two sets of plates; and it made no difference which of the ends of the wires of the helix were connected to the line-wires, or whether the currents entered the wires of the belix in the same or in opposite directions.

The helix in the last case having been replaced by a condenser, the other arrangements remaining unaltered, speech was again transmitted with as much facility and clearness as if the circuit had been complete-the capacity of the condenser, which was one microfarad, being amply sufficient to allow conversation to be carried on.

XLVI. On Method in Causal Research. By $\mathrm{S}$. Tolver Preston*.

A POINTED reflection as to the "danger of forgetting A that mathematical reasoning can only lead to useful results when founded upon definite physical conceptions" $\dagger$, deserves, no doubt, to be fully indorsed; and nowhere perhaps could this remark be said to be more justly applicable than to the problem of the constitution of the æther, which may be regarded as underlying the basis of the science of optics. No one, of course, would for one moment underrate

* Communicated by the Author.

$\dagger$ 'Nature,' vol. xxi. p. 370 (letter of correspondent). 
the value of mathematics in physical inquiries. Its achievements, when resting on definite physical conceptions, may be illustrated by the splendid results of the kinetic theory of gases; its complete powerlessness, in the absence of such conceptions, may be equally well exemplified by the inquiry as to the constitution of the ather. Nowhere, perhaps, have the spiritualistic assumptions about "force" nately applied as here, to the complete exclusion of all that can be called clear physical conceptions. This has naturally resulted from the fatal step taken at the time of Newton, of putting forward that notion of "action at a distance," which he characterized as a "great absurdity," and out of which inevitably sprang the endless train of "forces;" of which it is aptly remarked by the writer of the article on "the Atomic Theory of Lucretius" (North British Review, March 1868, page 239):- "This idea of the constitution of matter was perhaps the worst of all. .... No attempt was made to show how or why the forces acted; but gravitation being taken as due to a mere 'force,' speculators thought themselves at liberty to imagine any number of forces, attractive or repulsive, or alternating, varying as the distance, or the square, cube, fifth power of the distance, \&c."

Sir W. Thomson observes on the waste of mathematical skill due to the speculations about "force," much in the following terms $\uparrow:$ - "The eighteenth century forms a scientific school of its own, where in place of the not unnatural assertion of the ancient schoolmen-' $a$ body cannot act where it is not,' the most extraordinary of all paradoxes was set up'contact does not exist.' This strange notion took deep root; and out of it sprang a barren tree, which exhausted the ground and overshadowed the whole field of molecular physics, and upon which so much useless work of the great mathe-

* It will perhaps be said that the notion of "force" (in the sense of an action across space without the intervention of matter) is now almost abandoned, at least in this country. But then there is a great difference between the mere tacit relinquishment of an assumption or theory and that practical indication of its complete rejection which is afforded by the stirring-up of the mind to active inquiry and realization of the physical conditions that can alone replace the assumption. That this result is far from being generally attained yet, more especially in regard to that which must be expected as to the functions of the æther, where the physical deductions inevitably following on the rejection of "force" would be of immense practical interest, I think I shall sufficiently prove in the sequel. Indeed it will become apparent that the former vast influence of the notion of " force" still remains almost undiminished in relation to the æther.

+ I translate conveniently from a German quotation (that I happen to have by me) from the work 'Reprint of Papers on Electrostatics and Magnetism,' 1872. 
maticians of the beginning of the nineteenth century has been expended."

Faraday, Davy, and Rumford are known to have opposed the notion of "action at a distance." It is thus refreshing to see that the greatest leaders of physical science, those who have added most to knowledge and contributed most to real progress, have always been averse to this kind of spiritualistic doctrine.

In the Report of the British Association for 1862 is a paper by Prof. Stokes, entitled "Report on Double Refraction" ", to which I refer here, as this paper gives an account serving well to illustrate some of the different theories of the æther. I would particularly beg of those who are singly interested in the advancement of knowledge, and who would desire to take an unbiassed and unprejudiced view of the case, to give a share of attention to the following points which I shall develop. I need not add that I do not enter on this subject lightly, having already devoted some years of study to the question. First the theory of Fresnel is mentioned; and the Report states of it (p. 253) that the rther "is regarded as consisting of distinct material points, symmetrically arranged, and acting on one another with forces depending, for a given pair, only on the distance."

Here, it will be observed, we have the arbitrary assumption about "force" (or "action at a distance"). It is stated further in the Report that "Fresnel distinctly makes the supposition that the æther is incompressible, or at least is sensibly so under the action of forces comparable with those with which we are concerned in the propagation of light." Prof. Stokes then raises the probably not unnatural question whether this supposition of "incompressibility is not inconsistent with the assumed constitution of the æther" (given above); and he adds (page 253), "I have mentioned these points, because sometimes they are slurred over, and Fresnel's theory spoken of as if it had been rigorous throughout."

The theories of Cauchy and Neumann are then referred to. It is stated that, according to their view (page 254), "the æther is supposed to consist of distinct particles, regarded as material points, acting on one another by forces in the line joining them which vary as some function of the distances; and the arrangement of these particles is supposed to be dif-

* My attention was directed to this Report by a reference to it by the late Prof. Clerk Maxwell, in the article "Ether" in the new edition of the Encyc. Brit., where it is also remarked-"The undulatory theory, in the form which treats the phenomena of light as the motion of an elastic solid, is still encumbered with several difficulties." 
ferent in different directions. The medium is further supposed to possess three rectangular planes of symmetry \&e."

We see that the same arbitrary speculations about "force" are applied here. Alluding further on to a particular result deduced by Cauchy, Prof. Stokes observes (page 259):-"This result is, however, in the present case only attained by the aid of two sets of forced relations; that is, relations which there is nothing à priori to indicate, and which are not the expression of any simple physical idea, but are obtained by forcing the theory, which in its original state is of a highly plastic nature, from the number of arbitrary constants which it contains." (The number of these arbitrary constants is further on stated to have been twenty-one!) Probably this may be sufficient to illustrate the waste of the highest mathematical skill on this subject. It is said that these speculations were inserted in the Exercices de Mathématiques; so that possibly as an exclusively mathematical study (apart from any physical applications) the labour may not have been altogether lost.

Prof. Stokes makes the remark (on page 262):- "The arguments in favour of the existence of ultimate molecules in the case of ponderable matter appear to rest chiefly on the chemical law of definite proportions, and on the laws of crystallography, neither of which of course can be assumed to apply to the mysterious æther."

I would venture to put the question here, whether the best argument for the molecular constitution of the ather (as of gross matter) is not to be found in the fact that the æther is observed to have certain properties, and that these properties would be totally inexplicable unless the æther were assumed to be molecular. This was the argument of Lucretius, adopted and expounded by Newton in regard to gross matter (and applies equally to the æther). We can only have recourse to arbitrary assumptions about "force," or to dogmatic affirmations, which exclude all possibility of an explanation, unless we regard the æther as molecular. In a paper "On the Dynamical Theory of Gases" (Phil. Trans. 1867, page 49), Prof. Maxwell has made a remark which $I$ have before had occasion to quote, viz.:- "The properties of a body supposed to be a uniform plenum [i.e. not molecular] may be affirmed dogmatically, but cannot be explained mathematically." It would follow from this that, unless the ather be inferred to be molecular, its properties can only be affirmed dogmatically, or no rational account can be given of properties that can exist solely in virtue of the explanation that underlies them.

In fact, if the spiritualistic notion about "force" had nerer 
been invented, it is difficult to see how it could have occurred to any one to imagine that the æther could be in any respect different from gross matter, excepting as to the scale or the degree of motion of its parts. For let it be noticed that the endless assumptions that have been made about the æether-as to its being essentially different from gross matter (as if there were two kinds of matter in existence), as to its being imponderable (as an occult quality) - and all the mysterious attributes that have been ascribed to it, rest in their totality upon the assumption of "force," and are therefore completely gratuitous, or of the invention of those who invented "force." If this notion of the existence of "force" had never been put forward, it could not fail to have been seen that the only conceivable way in which matter can be physically affected is by motion; and the supposed "laws" of nature (in the sense of mere arbitrary fiats, which are based upon no dynamical conditions, but upon the notion of "force") can in reality only be self-made "laws," grounded upon nothing else than upon the phantom "force" * The distaste that at present exists for the study of that magnificent physical agent the æther, may be no doubt largely attributed to the spurious mystery thrown over the subject by the occult notion of "force;" and this dislike for the study would probably disappear with this notion; progress would be rendered possible. The one fact of the æther forming the physical groundwork of the science of optics should surely be enough to exite interest in this agent.

The splendid results pictured by modern inductive reasoning, whereby the retber must be inevitably regarded, by the rejection of the occult notion of "action at a distance" (and by the light of the principle of the Conservation of Energy), as

* It would no doubt be unjust if the blame for these vagaries in regard to "force" were entirely attached to the present generation, without keeping fully in view the fact that it was incoulated by some of its precursors, who (in spite of the protests of Newton) imagined that without any addition to knowledge, but by the mere invention of a phrase ("force," "attraction," \&c.), they could render unnecessary the search for the cause of gravitation. Rumford's words of protest against this were, "Nobody surely in his sober senses has ever pretended to understand the mechanism of gravitation." Huyghens, Hobbes, Leibnitz, Descartes, (Newton himself), and all the leading men of that period, as Lange relates in his notable historical work Geschichte des Materialismus (vol. i. p. 264), were of the same view as Rumford. But not only (as justly remarked in this work) did those who invented "force" (or "action at a distance") bring forward nothing themselves, but (becoming numerically the stronger party) they checked progress by throwing cold water on the efforts of those who attempted to account in a rational manner for phenomena; and it has hence been a matter of considerable difficulty to attract a reasonable amount of attention to any explanation whatever of the effects of gravitation. 
the general source of the motions developed in gross matter* (in the coal of the steam-engine, the explosion of gunpowder, chemical action generally, gravity, \&c.), would come to be contemplated and talked of as worthy of the intense interest that such problems must excite in minds capable of appreciating their rational worth. Under the reign of spiritualism the simple physics of Newton and his contemporaries run the risk of being forgotten; and for the method and strict system that existed then, which was the very keystone to progress, we have the absence of method $\dagger$, resting upon the abortive and endless speculations about "force," whereby every thing is involved in mystery and the rational inquirer discouraged. How shall the magnificent proofs of a concealed store of motion exhibited by nature in the endless phases of motion developed in gross matter on every hand, ever assert themselves in the face of a spiritualistic theory (based on "action at a distance") $\downarrow$, viz. that energy can exist without motion (i. e. in some ghostly form), that energy can in fact have a duplex nature (kinetic and not kinetic) and exist in matter at rest? It is so far per-

* There would seem to be some who profess to reject " action at a distance," and yet do not recognize the only logical alternative that could replace it, viz. the fact that motions developed in gross matter must be derived from a material agent in space. Where is the motion to come from consistently with the Conservation of Energy, unless it come from an external material agent that encloses a store of motion? For it is needless to add that we can have no recourse to "force" or "potential" energy by the rejection of "action at a distance." The recoguition of the $f$ fat of the derivation of the motion from the material agent is obviously the necessary preliminary towards au inquiry after the process. How shall progress ever be possible unless these facts are first debated and realized? Shall a truth wait for appreciation until it becomes a truism or platitude. The time may come when the present superficial theories, whereby motions are referred en bloc to spiritualistic stores of energy (represented by the high-sounding phrase "potential" energy) will be looked back upon with something like contempt. It only needs that attention should be thoroughly roused to this matter to ensure this end.

$\dagger$ The grand fatality of the error of "action at a distance" consisted in its departure from a method; and errors of principle are always the most disastrous.

I The prevalence, even now, of the theory of "potential" energy (in the sense of an energy which is not kinetic), or the idea that energy is of two kinds, is an additional proof of the fact before stated, viz. that although "action at a distance" may be proessedly rejected, the logical consequences of its rejection are not adequately realized. There would not be such an objection to the term "potential energy" if it were not inevitably contrasted with kinetic energy as something different, thereby serving as a continued support to the fiction of " action at a distance." Indeed the prefix "kinetic" itself (applied to energy) acts as a perpetual cover to this fiction; for where would be the use of the distinctive prefix "kinetic," unless it were thereby implied that some other energy than "kinetic" energy (or the energy of motion) existed?

Phil. Mag. S. 5. Vol. 9. No. 57. May 1880. 
fectly certain (as has been proved again and again in science) that no truth is safe from being disguised by spiritualistic theories of this character, in the face of which reason loses its influence, and legitimate proof its power of appeal.

It does not seem to be adequately realized that there can be nothing mysterious in the mere scale of the parts of the matter that forms the rther, that it is as easy to reason of matter of one scale as of another, that smallness of moving particles (so as to allow number in unit of volume) is the essential condition for the concentration of the greatest possible amount of energy in a given volume of the ether, that it may be proved definitely beforehand that this energy, if it existed, would certainly be concealed in its normal state. All this should excite interest in the case. But what do we find in fact? The subject is scarcely discussed; and even care would not seem to be taken to inquire what we should expect to find if these things were true*. The mystery due to the notion of "force," which precludes all hope of any addition to knowledge, has rendered the subject repulsive.

May we not at least attempt what preliminary steps can be made towards an advance by dropping the spiritualistic assumption about "force." In the first place, as before remarked, we must regard the æther as of molecular constitution, or all means of accounting for its properties would be excluded. The most important observed fact in regard to the xther is perhaps the entire absence of any measurable resistance opposed by it to the passage of bodies (such as the

* No doubt the preconceived idea would naturally be that, in order for a motive agent to contain a store of motion adapted to produce such an effect as an explosion of gunpowder (for example), something boisterous and appealing to the senses would be required, not the thin impalpable æther whose very presence eludes direct detection by the senses. But on the question being examined into, exactly the reverse of all this found to be true. For it may be proved, with mathematical certainty, that the greater the intensity of a store of motion in the material agent, the less would it appeal to the senses. For the greater the velocity of the particles of a material agent, the less is the resistance opposed by the agent to the passage of masses through it, or the more impalpable does the agent become. This one fact, as a definite mechanical truth (independently of all questions as to its possible application in nature), ought surely to be enough to excite inquiry and arouse a rational interest. The vague and utterly unpractical way in which this great physical agent (the æether) is commonly treated would indicate an absence of realization of the physical qualities that we should expect to find in the æther if it were a great dynamic agent of enormous practical importance in the everyday operations of nature. If it were merely attempted (as an ordinary engineering problem) to reason out beforehand what qualities a material agent ought to possess in order to be adapted as a general source of motion, then the qualities the æther appears to have would be seen to be precisely of the kind required. 
planets for instance) through its substance. A molecular medium with its constituent molecules at rest, or in fixed positions (like a solid), could not conceivably be capable of satisfying this condition of free passage. Hence we must infer that the molecules (or atoms) of æether in their normal state are perfectly free to move past each other without obstruction; for on no other condition could the ather yield freely to a passing body. Next, we must infer that the atoms of xther are in motion in their normal state. For, first, this inference is necessary on the ground that a medium with atoms at rest, and with no power of acting on each other at a distance, would have no positive properties at all. Secondly, another independent reason for this inference is that, if the atoms were at rest, they would unavoidably be put in motion by a body passing through the ether, which would entail some appreciable resistance-which is contrary to observation. The only means of avoiding the resistance to passage is for the atoms of æther to be already in motion, whereby the transference of motion by the moving body to the æther atoms in front may be (sensibly) compensated for by the transference of motion (conversely) from the æther atoms in rear, to the body. If on the other hand, the ather atoms in rear of the body were not already in motion, they could not of course transfer any motion to the passing body. Indeed it has been proved mathematically with perfect definiteness, that the greater the velocity of motion of a system of atoms, the less is the resis- tance opposed by the medium (formed of these atoms) to a body passing through it; so that by an adequate velocity for the atoms, the resistance will be rendered inappreciable. This, therefore, affords a complete means of explaining the observed absence of appreciable resistance to bodies passing through the æther. Next, considering therefore the æther as consisting of atoms in free motion among each other, the question arises as to what is the character of the motion. It will be evident that atoms in free motion, and incapable of acting on each other at a distance, can only move in straight lines. Hence the inference that the æather is in principle a gas (constituted according to the kinetic theory) would seem to suggest itself at the very outset as an apparently inevitable deduction. No postulates whatever need be resorted to as to the general directions of the motions of the ether atoms among themselves, as that is all automatic, and dependent on principles already investigated in connexion with the kinetic theory of gases. The store of enclosed energy, the pressure, the density \&c., are then all connected by rigid dynamical relations, not in any way permitting of arbitrary speculation. As regards $2 \mathrm{D} 2$ 
a possible insight that this constitution of the æther might afford into the transverse vibrations of light, and the effects of gravity (under Le Sage's sheltering principle), by admitting that the atoms have an adequately long mean path-I must refer to a previous article * in 'Nature' vol. xxi. page 256, and also Phil. Mag. Sept. \& Nov. 1877.

At least it appears to the writer that to explain the observed absence of resistance to the passage of bodies through the wther is very important. It is difficult to imagine what is supposed to take place in these "statical" theories, where the æther is supposed to consist of material particles in fixed positions and assumed to be acting on each other by "forces" at a distance, or the constitution of the rether is regarded as resembling that of a "solid." What becomes of the delicate structure of particles and "forces" in the "solid " with (say) the immense mass of the earth flying through the "solid" at about 18 miles per second? Can even the fertile resources of these plastic theories, when taxed to their utmost extent, be of avail here? The objection is not the less cogent because elementary.

There would seem to be sometimes a certain looseness of reasoning or carelessness in regard to the treatment of the xther, that would be considered out of place in ordinary mechanical problems. Sir John Herschel, whose expositions are known in general to have been distinguished for their clearness and lucidity, seems to have made an exception in the case of the æther. In referring to the speculation as to the æther being a "solid," he ("Popular Lectures,' page 285) defines the solidity of the rether in the sense, that none of its elementary molecules are to be supposed capable of interchanging places $\dagger$, or of bodily transfer to any measurable distance from their own special and assigned localities in the universe." What (one might ask) becomes of the molecules of xther incapable " of bodily transfer from their own assigned localities," with a planet careering through them, or the solar system in its proper motion of several miles per second through space?

The vague and loose reasoning occasionally applied to the æther, may perhaps be partly accounted for from the feeling of utter hopelessness as to making any advance when all efforts are paralyzed by the boundless labyrinth of speculation involved in the assumption of "force." Can it be seriously believed

* The proposed mode of accounting for the transverse vibrations of light, given in the article in 'Nature,' was based on a suggestion or hint thrown out by Prof. Clerk Maxwell, under subject " Ether" (Encyc. Brit. new edition).

$\dagger$ The italics are Sir John Herschel's. 
for one moment that this complete helplessness is in truth a necessary thing, or that it is any thing more than the natural consequence of a single departure from the strict and defined path of reason, by making an assumption without a definite conception to base it upon? A single false step may well be fatal if it involves the departure from a system (or the overthrow of a guiding principle).

No doubt the above theory, suggested as a groundwork for the constitution of the æther, will, from its very definiteness, be specially liable to attack, since there will not be the power of "saving appearances," by introducing first one hypothetical "force" and then another, that belongs to the plastic statical theories. My object, however, has not been so much to suggest a theory as to point to the necessity for a system in order to find one. Difficulties may naturally be expected to present themselves at first. If, however, the theory suggested were only capable of explaining a single fact, such for instance as the absence of resistance of the rether to the passage of bodies, then even this might fairly be regarded as more satisfactory than those dogmatic assumptions that exclude all possible explanation so long as they are adhered to. The theory at least shows the æther to be a body necessarily uniform as to density and pressure, and enclosing a store of concealed energy of probably enormous value in a small volume of space, this latter quality being precisely that required to render the æther fitted to be a powerful motive agent, capable of playing a most important part in the everyday operations of nature. Who shall estimate the practical (I might say the industrial) value of a study that deals with the source of the motions developed in gross matter generally? and, since knowledge itself is power, who can positively say beforehand how far a clear realization of the existence of these exhaustless stores of motion may go towards their possible practical utilization under more advantageous conditions?

But the universal recognition of the immense power of a method gained by the complete renunciation of " force," would probably be of far more value than the discovery of any concrete facts, since the appreciation of the worth of a method may afford the indispensable key to a science of discovery. That no method or system exists, and therefore that the essential condition for a successful research into the hidden nature of physical causes is entirely wanting, so long as the spiritualistic notion of "force" is adhered to, is at least an absolute certainty, of which any one can convince himself without much thought, and that independently of taking the trouble to inquire into the legitimacy of the assumption of 
"force." For where, indeed, would be the gain of inquiry into the legitimacy of that which, when put forward as a cause, serves only to make phenomena darker than before, by attributing occult qualities to matter, and, in addition to this, leaves one without a scientific method, entailing a fruitless waste of intellectual energy. To those who might say that I have assumed the idea of "force" to have more influence than it actually has, I reply:-Recognize, then (for the sake of consistency), the sole logical alternative that can replace it, viz. the fact that the ether (or material agent in space) is the source of the endless phases of motion developed in gross matter on all sides-since the recognition of this fact is the only proof that can be afforded that "force" (with its attendant spiritualistic stores of energy) is rejected, not in profession, but in practice. After the recognition of this fact, then the inquiry into the mode or process involved in the derivation of the motion from the material agent, becomes the next step, which presents itself as an engineering problem of great practical interest.

I venture to think that the critical remarks made in this essay will not be considered inopportune. May it not well be conceivable that the immense success of experiment in this age has caused the value of a strict and pure system of theoretic reasoning to be less appreciated than would otherwise have been the fact? or that the danger of wandering from such a system is greater (because its effects are less felt) when we have the resource of experiment to fall back upon? The ancient Greeks, who had not recognized the worth of experimental research, were consequently thrown back upon theoretic reasoning as a sole guide; and the value of a strictly rational system was every thing to them; and the remarkable progress they made in just theoretic views as to natural facts is admitted (some of their fundamental deductions remaining unshaken to this day). One may well imagine the confusion that would have resulted, had the notion of "force " and the attendant wandering into a maze of spiritualism, occurred to them, without the backstay of experiment to put a curb on these vagaries.

Nevertheless, although the w'orth of experiment can scarcely be overestimated, it is no doubt none the less true that the general agreement upon a uniform and strict theoretic system would be of immense practical value as an accompaniment to this. Indeed, when one compares the enormous advantage we possess in the present day in the extensive knowledge of facts accumulated through years of experiment, with the disadvantage the ancients laboured under in their dearth of know- 
ledge in this respect, one may be almost led to surmise that the progress made in the present day in obtaining an insight into the hidden nature of physical causes has not kept pace with this increased knowledge of facts; and this would hint again at the evil attendant on the want of a method. Also the lull of any great discoveries in the field of pure physies during the last few years would seem to make the direction of attention to any fresh pathway all the more opportune.

London, April 1880.

XLVII. On Berthelot's Thermo-Chemistry. By J. P. Cooke, Jun.*

TTHE new work of M. Berthelot, entitled Essai de Mécanique

Chimique fondée sur la Thermo-chimie,' presents for the first time in a systematic form the results accumulated during the past ten years from one of the most fruitful fields of investigation ever opened to the chemist. The book supplies a most important want; for the details of the work, published in numerous separate papers rapidly following each other in the chemical journals, have been almost unintelligible, except to those who have followed the investigation from the beginning, and no connected statement of the general principles involved was accessible to the student. The work in this new field has been done almost wholly by two investigators-Berthelot, of Paris, and Thomsen, of Copenhagen. Guided by different theoretical views, these skilful experimenters have gone over very nearly the same ground; and their united testimony, concurrent as it is in most cases, gives a certainty to the results obtained which is as fortunate as it is unusual when the field explored is so extensive as the one we are considering. These two men alone could write authoritatively on the subject; and it is, perhaps, fortunate that the first presentation should come from M. Berthelot, who has the asual skill of his nation in exposition and generalization.

In his introduction, Berthelot enunciates the fundamental principles of thermo-chemistry under the three following heads:-

\section{Principle of Molecular Work.}

I. The quantity of heat evolved is the measure of the sum of the chemical and physical work accomplished in any reaction.

\section{Principle of Conservation of Energy.}

II. When a system of bodies, simple or compound, starting from a given condition undergoes either physical or chemical

* From Silliman's American Journal, April 1880. 